\title{
Comparing and Analysis on Adaptive Information-brokerage Schemes in Wireless Sensor Networks
}

\author{
Xing-Po $\mathrm{Ma}^{1 *}$, Guo Song ${ }^{1}$, Lei $\mathrm{Li}^{1}$, Yan-Ge Sun ${ }^{1}$ and Jun-Bin Liang ${ }^{2}$ \\ ${ }^{1}$ School of Computer and Information Technology, Xinyang Normal University, \\ Xinyang 464000, P.R. China \\ ${ }^{2}$ School of Computer and Electronics Information, Guangxi University, Nanning \\ 530004, P.R. China \\ ${ }^{1}$ maxingpo@xynu.edu.cn
}

\begin{abstract}
In wireless sensor networks, how producers route and store their own data and how consumers retrieve the desired data efficiently are collectively known as the informationbrokerage problem, which is challenging because of the short communication radius and the limited energy of the sensor nodes. It has been shown in existing works that selecting some sensor nodes as the rendezvous nodes to both store the data and reply to the queries from the consumers is a feasible way to solve such a problem. However, when selecting the optimal rendezvous nodes, most of the existing schemes do not consider the following two factors: the event data generating rates of the producers and the query frequencies of the consumers. In fact, those two factors are very important when determining the optimal locations of the rendezvous nodes because of the dynamic characteristic of the sensor networks. As far as we know, there are mainly three schemes which consider the abovementioned factors when determining the rendezvous nodes. The three schemes are named VRS, MODS and ODS respectively, where VRS is our former work. In this paper, we modify VRS and add some important algorithms to make it much more perfect. Moreover, we compare the three schemes through extensive simulation, and make reasonable analysis on the simulation results. It is hoped that our work can be a valuable referee for the researchers on solving the information-brokerage problem.
\end{abstract}

Keywords: Wireless sensor networks; adaptive information brokerage; compare; analysis

\section{Introduction}

A sensor network is consisted of a large number of sensors which can acquire useful information (e.g., photic, thermal, acoustic and stressful data) from the physical environment around. There are many kinds of applications for the sensor network, from environment monitoring to scientific data collection, to medical use and military applications. In some applications, the sensor network can be seen as a "distributed database". That is, it stores the sensing data in a distributed way and can response for the query from any points in any time in the sensor network. e.g., in a zoo, in which a lot of sensors are deployed, sensors can collect and store the data of the animals, and the tourists at any place in the zoo can send a query to the sensors to get the information of the animals. In this new kind of "database", one of the important query is event-based query $[1,2]$, e.g. " "what has happened in the network recently?" In those applications, the event data producers should know where to store the data and the consumers who are interested in the event data should know how to retrieve the data.

\footnotetext{
* Corresponding Author
} 
The problem mentioned above can be formulated as the information-brokerage problem, which specifies how to route, store and retrieve the data. To solve this problem efficiently, a good data storage model should be constructed in wireless sensor networks.

Because the sensor networks are dynamic, the data storage model should be adjusted adaptively according to the change of the network status. In this paper, the nodes which are embedded in the data storage model are called storage nodes or rendezvous nodes. Generally speaking, the rendezvous nodes should be as close to the nodes that generate the most sensing data or retrieval requests as possible to decrease the energy consumption on data storage and retrieval. Thus, two factors must be considered while adjusting the network model or the rendezvous nodes: the sensing data generating rate of each producer and the query frequency of each consumer in the sensor networks. However, at present, only a few of the existing schemes on data storage and retrieval have considered the two factors. As far as we know, there are mainly three such schemes: VRS [3], ODS [4] and MODS [5].

VRS is a virtual-ring-based scheme, which was proposed by us in 2012. Although the main idea of the scheme VRS has been presented in [3], some related algorithms are not included in [3] because of the paper length. We make VRS more integrated by adding the related algorithms in this paper. MODS is a relatively new scheme which was proposed in 2015. Thus, it is needed for us to do more experiments to compare the performances of the three schemes mentioned above, and find out the best one. In brief, the main contributions in this paper are as follows:

- The scheme VRS is presented in greater detail. We add two more important algorithms in this paper as the supplements in [3]. Specifically, the added algorithms are Algorithm 1 in sub-section 3.3.3 and Algorithm 2 in sub-section 3.3.4.

- Extensive experiments have been done to compare VRS, ODS and MODS. Moreover, the analysis on the simulation results is also given.

The arrangement of this paper is as follows. Section 2 introduces the related work; Section 3 presents the overview of the schemes ODS, MODS and VRS. Moreover, more details of VRS with two added algorithms are also presented in this Section; Section 4 illustrates the simulation results with detailed analysis; Section 5 concludes the paper.

\section{Related Work}

To solve the problem of information brokerage in wireless sensor networks, existing works mainly follow two basic approaches. One of which is data-centric routing [6], and the other one is data-centric storage [7].

The earliest two works that adopt the data-centric-routing-based method are Directed Diffusion [6] and TinyDB [8]. Directed Diffusion floods the queries across the sensor network. When the desired data are met by the queries, they will be sent back to the consumer following some kind of enhanced routes. It is clear that Directed Diffusion will not be effective when the queries launched by the consumers are frequent. Similarly, in TinyDB, sensor nodes store their own data locally, and the consumers flood the queries across the whole sensor network to find the desired data. Another data-centric-routingbased scheme, which is proposed for data storage and retrieval, is Rumor Routing [9]. Rumor Routing achieves data storage and data retrieval by random routing. Specifically, on the one side, any data producer in the sensor network sends the data in a random direction, and the nodes that are along the route that the data follow will also store the data. On the other side, any data consumer will generate a query and also forward the query in a random direction. The consumer will find the desired data if the forwarding route of the query intersects with the forwarding route of the desired data. Clearly, Rumor Routing can't guarantee high successful retrieval rate, and it is not effective because of the random forwarding. 
For the approach based on data-centric storage, the data generated by the producers is left on the rendezvous nodes for consumers to retrieve. The geographical hash tables (GHTs) [10] is a classic work in this category. One of the short comings of GHTs is that it brings a hot spot problem. That is, the rendezvous nodes and the nodes around the rendezvous nodes take much more load, compared to other nodes. To solve this problem, a lot of work has been done later.

DBAS [11] and DCAAR [12] hash an event type or an attribute of an event type to a grid region, instead of a point. EHS [13] hashes an event type to a point in a face, and the nodes in the face are responsible for the data storage and retrieval. Reference [14] applies diffuse caching on top of DCS to solve the problem of hot spot. Another problem in GHTs is data retrieval may be failed when the nodes near the hashed points move. Reference [15] proposes a cluster-based approach named C-DCS to solve this problem. Moreover, reference [16] presents a data centric scheme which aims to make the storage space of the sensor nodes evenly.

Some works on the problem of information brokerage combine the approach of datacentric routing and data-centric storage, e.g., References [17] and [18]. All the works mentioned above do not consider the data rates of the consumers and data-retrieval frequencies of the producers while choosing the rendezvous nodes. As far as we know, there are mainly three schemes, namely VRS [3], ODS [4] and MODS [5], which consider the above-mentioned two factors. VRS is our former work, and it is based on a virtual ring model which can be adjusted according to the data rates of the producers and the data-retrieval frequencies of the consumers in wireless sensor networks. ODS achieves adaptive data storage and retrieval by adjusting the only rendezvous node adaptively according to the dynamic characteristic of the sensor networks. MODS is similar with ODS, and the main difference is that MODS chooses multiple sensor nodes as the rendezvous nodes.

As far as we know, there have been no articles which compare performances of the three above-mentioned schemes. Thus, we do such work in this paper.

\section{Adaptive Information-brokerage Schemes}

\subsection{The ODS Scheme}

In ODS [4], one sensor node is selected out of set $\mathrm{V}=\left\{v_{1}, \ldots, v_{N}\right\}$ to act as the rendezvous node according to the data generating rates of the producers, the dataretrieval frequencies of the consumers and the location information of all the sensor nodes, where $v_{i}(0<i \leq \mathrm{N})$ in $\mathrm{V}$ denotes the sensor node whose ID is $i$. All producers send their data to the rendezvous node to get stored, and all consumers retrieve the data which they are interested in by sending their data-retrieval requests to the rendezvous node. The rendezvous node in ODS is determined by finding out which node can minimize the total communication cost of all the sensor nodes on data storage and retrieval when the node acts as the rendezvous node. Moreover, the rendezvous is selected periodically to go with the dynamic change of the network status.

\subsection{The MODS Scheme}

In ODS [4], one sensor node is selected out of set $\mathrm{V}=\left\{v_{1}, \ldots, v_{N}\right\}$ to act as the rendezvous node according to the data generating rates of the producers, the dataretrieval frequencies of the consumers and the location information of all the sensor nodes, where $v_{i}(0<i \leq \mathrm{N})$ in $\mathrm{V}$ denotes the sensor node whose ID is $i$. All producers send their data to the rendezvous node to get stored, and all consumers retrieve the data which they are interested in by sending their data-retrieval requests to the rendezvous node. The rendezvous node in ODS is determined by finding out which 
node can minimize the total communication cost of all the sensor nodes on data storage and retrieval when the node acts as the rendezvous node. Moreover, the rendezvous is selected periodically to go with the dynamic change of the network status.

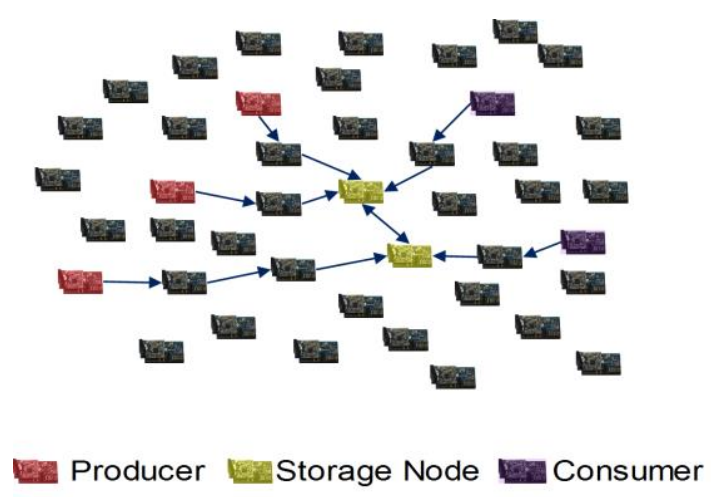

Figure 1. Data Storage and Retrieval in MODS

\subsection{The VRS Scheme}

3.3.1. Overview of VRS: We show the main idea of VRS as follows. At first, the whole sensor network field is divided into virtual rings (In the case of a square field, we divide the region that is surrounded by the inscribed circle of the square into virtual rings. The specific way of forming virtual rings can be found in [3]). The width of the virtual rings is $R$, which is also the communication radius of the sensor nodes. Then, one of the virtual rings is selected out to mainly take responsibility for data storage and retrieval. We call the selected ring the rendezvous ring. After that, all event data will be sent to the rendezvous ring to get stored, and all queries will also be sent to the rendezvous ring to retrieve the desired data. The virtual ring model in VRS is demonstrated in Figure 2.

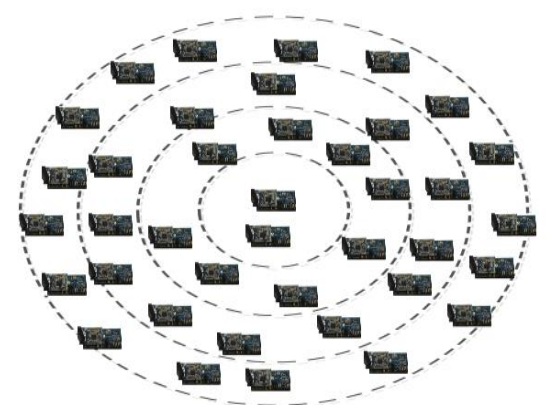

(a)

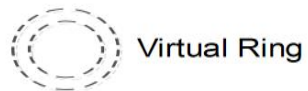

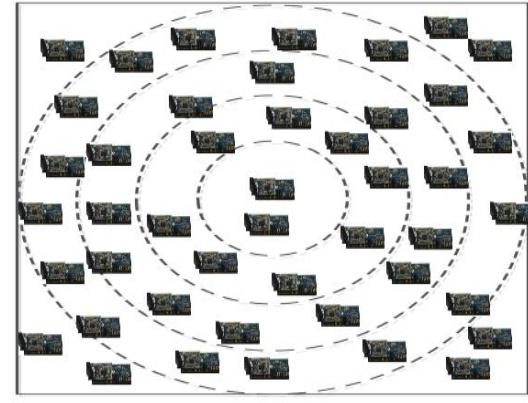

(b)

aㅐ를 Sensor Node

\section{Figure 2. (a) Virtual Ring in a Round Sensor Field; (b) Virtual Ring in a Square Sensor Field}

3.3.2. Procedure of data storage in VRS: In VRS, all the event data produced by the producers will be sent to the rendezvous ring. They will be stored by the nodes they pass by when they are sent to the rendezvous ring, and finally rest in the rendezvous ring. To achieve this, any producer whose ring ID is bigger than the ID of the rendezvous ring needs to route the data towards the center of the whole sensor network field using GPSR routing protocol [21], while any producer whose ring ID 
is smaller than the ID of the rendezvous ring must route the data away from the center. Suppose ring $\mathrm{k}$ is the rendezvous ring. If the ring IDs of the producers are smaller than $\mathrm{k}$, to send the event data to ring $k$, the destination of the event data packet can be set to any position whose distance to the center of the whole field is bigger than $(k+1) \times R$. This is because, in this case, the data packets will be intercepted by the rendezvous ring when they are being routed to their destinations. However, in VRS, to reduce and balance the energy consumption, the destination chosen for a producer whose ring ID is smaller than $k$ should satisfy another two conditions:

I : The destination, the producer and the center of the whole region are in the same line.

II : It should be on the same side with the producer against the center of the whole region in the line.

Algorithm 1 is given below to show the procedure of data storage in VRS.

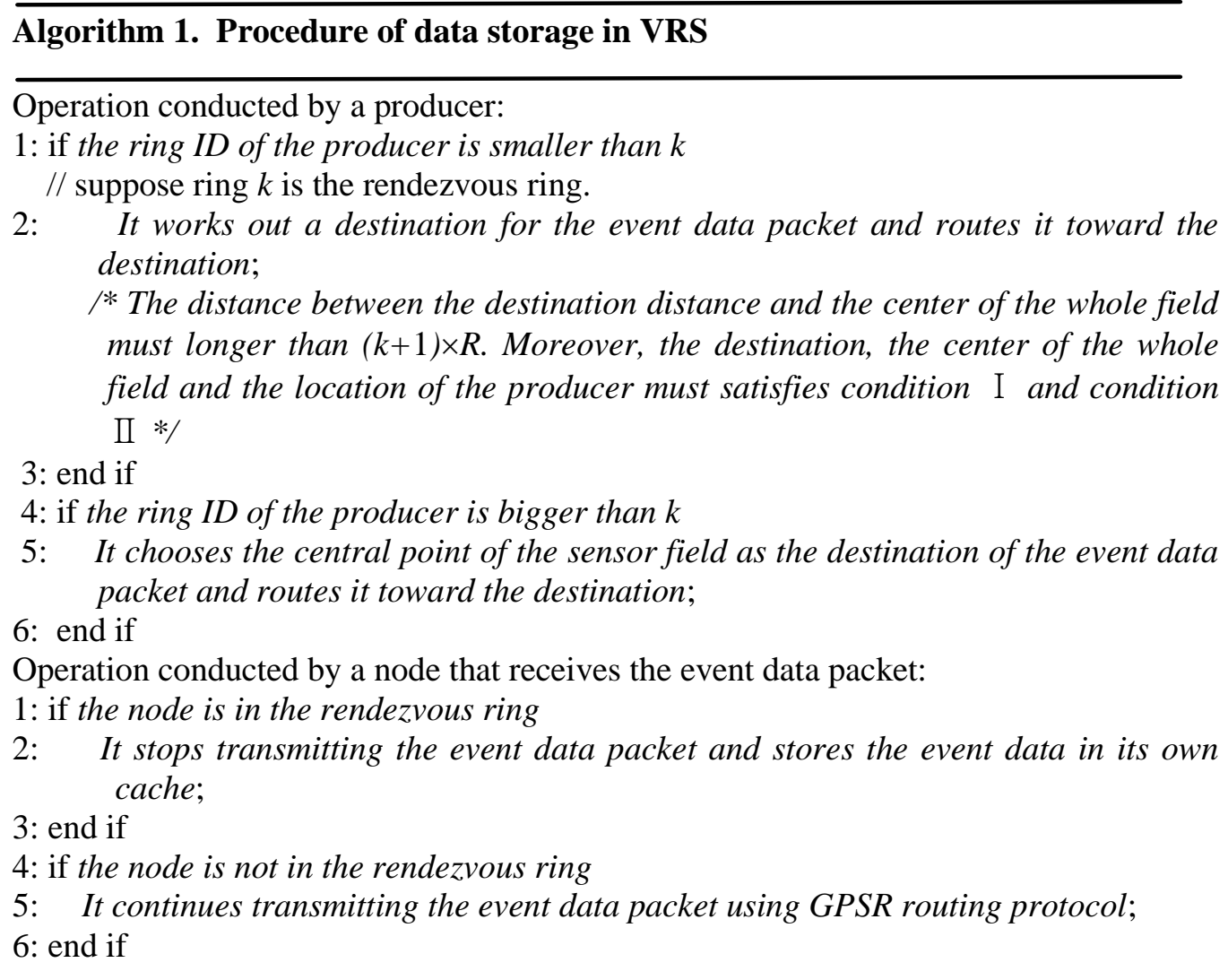

3.3.3. Procedure of data storage in VRS: In order to retrieve the desired event data, data-retrieval requests should be sent toward the rendezvous ring, because a copy of each event data packet is left in the rendezvous ring.

To discover the desired event data, the consumer generates a query packet and sends it to the rendezvous ring. Two cases should be considered. The first case is that the consumer is out of the rendezvous ring, and the second case is the consumer is in the rendezvous ring. If the consumer is out of the rendezvous ring, it should send the query to the rendezvous ring first, and then the query will be treated by the nodes in the rendezvous ring. Figure 3 illustrates the procedure of data retrieval in VRS, and the specific query-processing procedure in the rendezvous ring in given in Algorithm 2. 


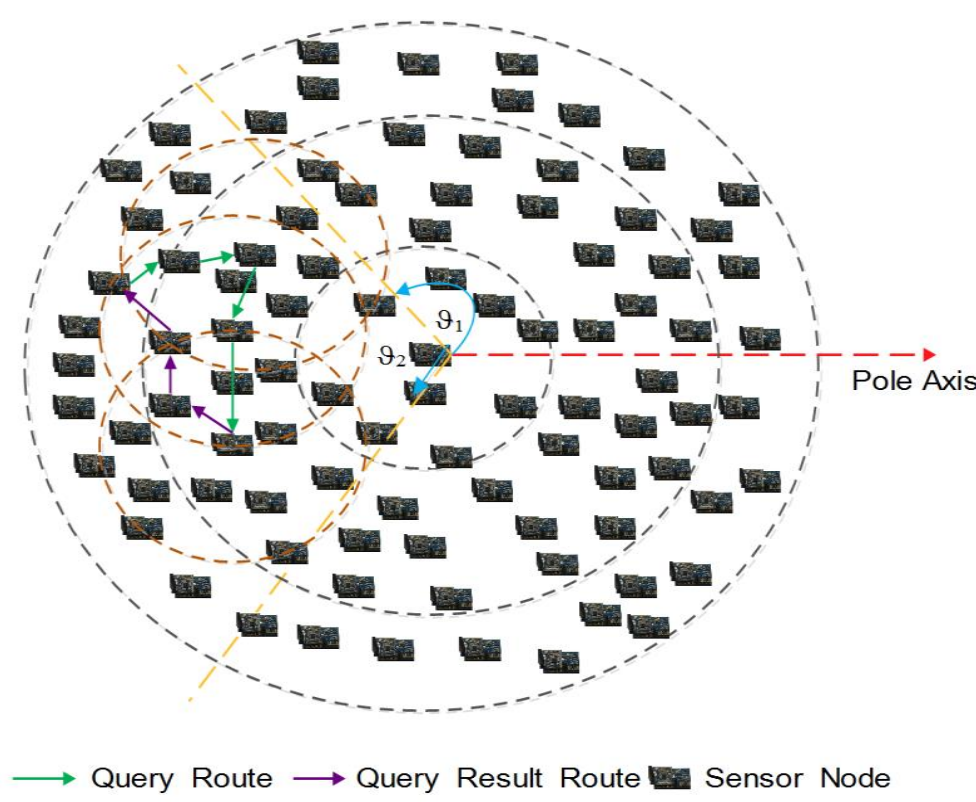

Figure 3. Procedure of Query Processing in VRS

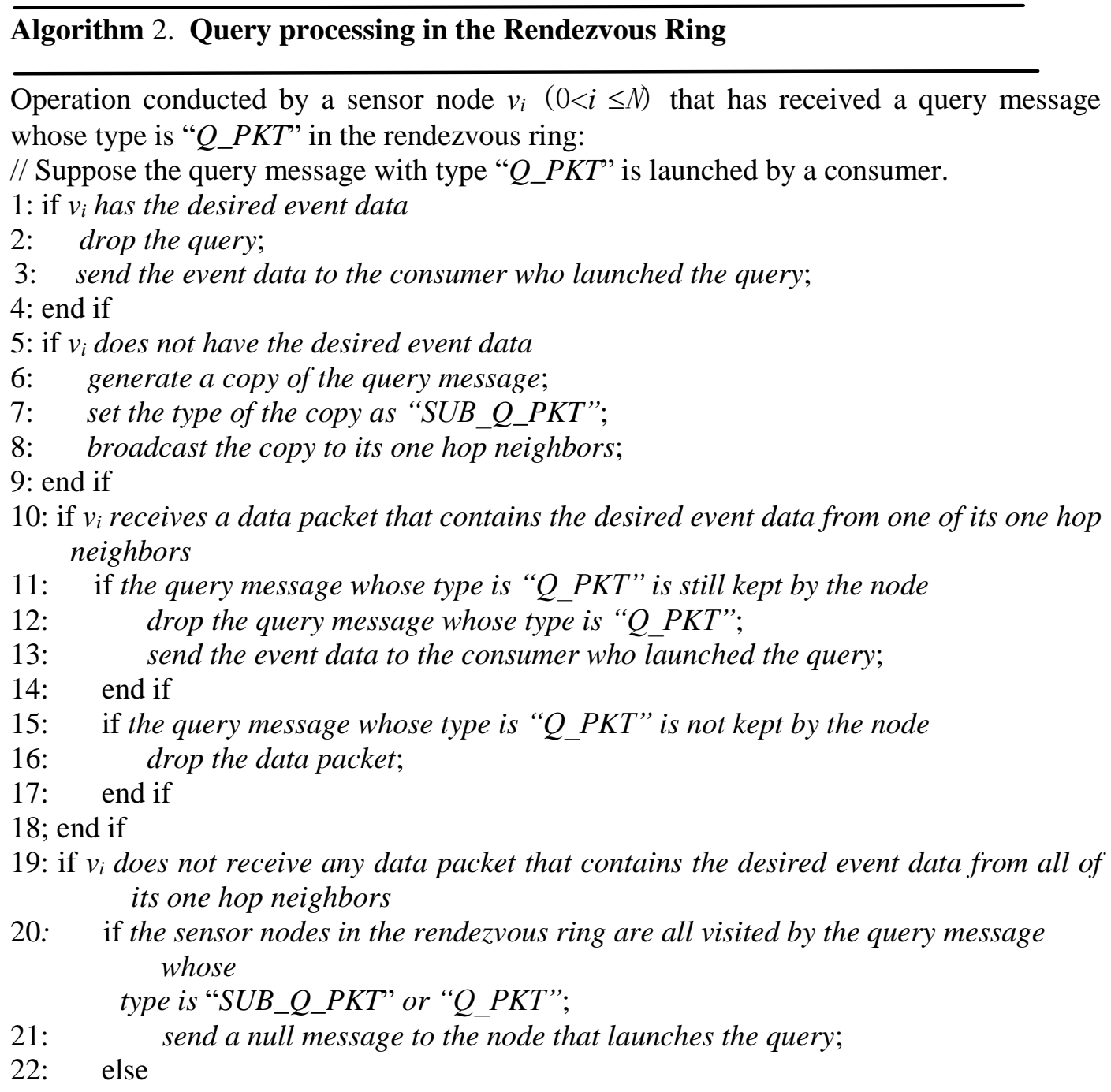


23: $\quad$ choose its farthest neighbor node counterclockwise in the rendezvous ring as

21: $\quad$ send the query message to the next destination;

22: $\quad$ end if

23: end if

Operation conducted by a sensor node $v_{i}(0<i \leq M)$ that has received a query message whose type is " $S U B \_Q \_P K T$ " in the rendezvous ring

1: if $v_{i}$ has the desired event data in its cache

2: Send the event data to the node that sends the query message whose type is "SUB_Q_PKT" to it;

3: end if

4: if there is no such desired event data in $v_{i}$ 's cache

5: $\quad$ send an empty message to its leading node;

6: end if

Table 1. Part of the Simulation Parameters

\begin{tabular}{|l|l|}
\hline Parameter Description & Value \\
\hline Size of event data package $\left(s_{d}\right)$ & 20 byte \\
\hline Size of query package $\left(s_{q}\right)$ & 10 byte \\
\hline Event data compression ratio $(\alpha)$ & 0.5 \\
\hline Maximum packet generating rate $\left(r_{\max }\right)$ & 1 packet every $5 \mathrm{~s}$ \\
\hline Radio transmission range $(R)$ & $40 \mathrm{~m}$ \\
\hline Transmitting cost of one byte data & $0.0144 \mathrm{~mJ} / \mathrm{byte}$ \\
\hline Receiving cost of one byte data & $0.00864 \mathrm{~mJ} / \mathrm{byte}$ \\
\hline
\end{tabular}

\section{Simulation}

We use OMNET++ as our simulator to evaluate the performance of VRS on realtime data discovery. We compare VRS with ODS and MODS mainly on the performance of network life time [19], the delivery latency [20] of data storage and retrieval, and the average energy consumption of the sensor nodes as well as the load balancing of the network.

\subsection{Simulation Set Up}

The experiment scenario is a $400 \mathrm{~m} \times 400 \mathrm{~m}$ square field, in which $400-900$ sensor nodes are deployed randomly. Part of the parameters in our simulation is listed in Table 1. Moreover, each sensor node chooses two numbers from range $\left(0, r_{\max }\right)$ randomly as its event-data-packet-generating rate $r_{d}^{i}$ and query-packet-generating rate $r_{q}^{i}$ respectively. In MODS, the number of the rendezvous nodes is set to 10. We use GPSR [21] as our routing protocol to route a data packet from one point to another. Of course, if WSNs are deployed in a underwater background, routing protocols such as that proposed in [22] can be used. In the following of this paper, the effective time of a data item refers to the time length from the time point when a sensor node begins to store the data item to the time point when the sensor node begins to mark it as an outdated data item. 


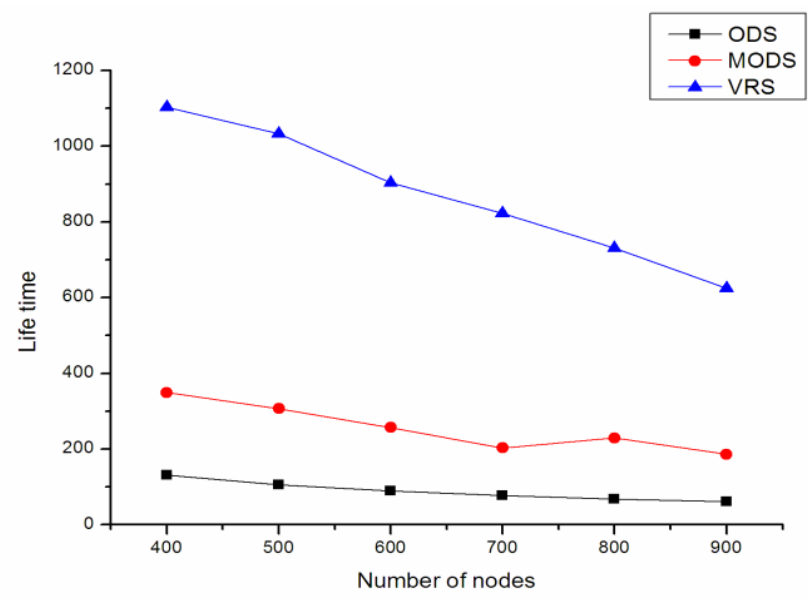

(a)

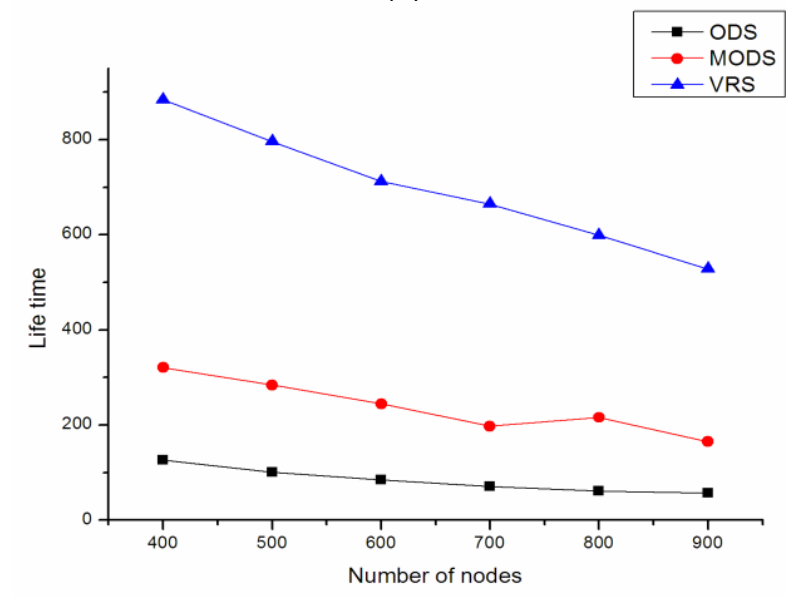

(b)

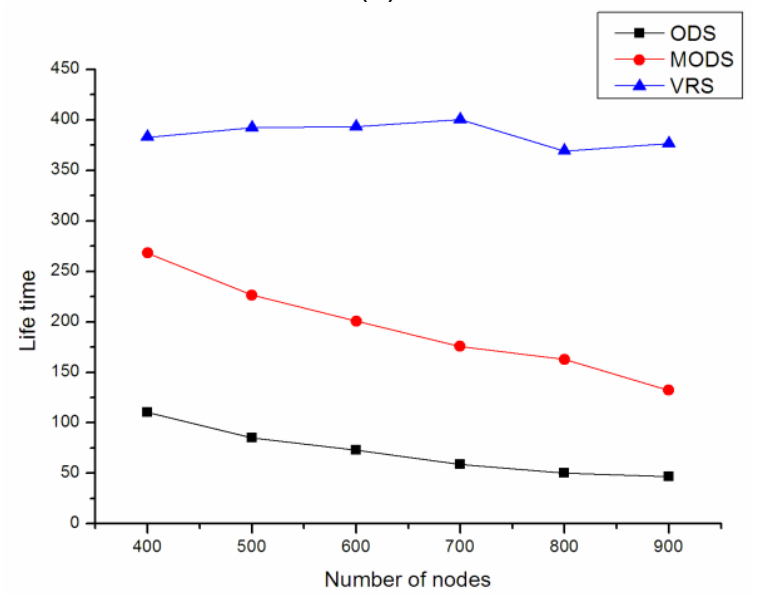

(c)

Figure 4. Performance of the Life Time: (a) effective time $\tau=1 \mathrm{~s}$; (b) effective time $\tau=0.5 \mathrm{~s}$; (c) effective time $\tau=0.2 \mathrm{~s}$

\subsection{Performance on Life Time}

In this section, we show the simulation results about the life time [20] of the sensor network in ODS, MODS and VRS. In this experiment, the initial energy is set to $1 \mathrm{~J}$, and the node number is set from 500 to 900 respectively. 
Figure4 shows the life time of the sensor network in ODS, MODS and VRS with different data effective time $(\tau)$. We can see that, in most cases, the life time of the three schemes all decrease as the node number increases. This is because, as the node number of the sensor network increases, the load of the nodes in the rendezvous ring also increases. We note that the life time of the sensor network in VRS does not change too much as the node number increases in Figure $4(\mathrm{c})$. The reason is that, when the data effective time is small enough, almost all the queries should come into the rendezvous ring to meet the event data. As the node number increases, the chance for a query to meet the desired event data with a small hop count in the rendezvous ring increase, and this may decrease the energy consumption on query processing in the rendezvous ring. However, the cost of the nodes on storing the event data may increase as the node number increase. Thus the total energy consumption of the sensor nodes in the rendezvous ring does not change too much, and this makes the life time not change too much.

We can see that, as the data effective time decreases, the life time of the sensor network in the three schemes decreases either. The reason is, as the data effective time decreases, the query needs to go much farther to meet the desired event data, and this increases the energy consumption of the event data retrieval. However, the energy consumption of the event data storage does not change as the data effective time changes. Thus, as the data effective time decreases, the total energy consumption increase, and the life time of the sensor network becomes shorter.

It is clear that the life time of the sensor network in VRS is much higher than that in ODS and MODS, since VRS balance the load much better than ODS and MODS. Later on, we will show the simulation results about the load distribution of the sensor nodes.

\subsection{Performance on the Delay of Data Storage and Retrieval}

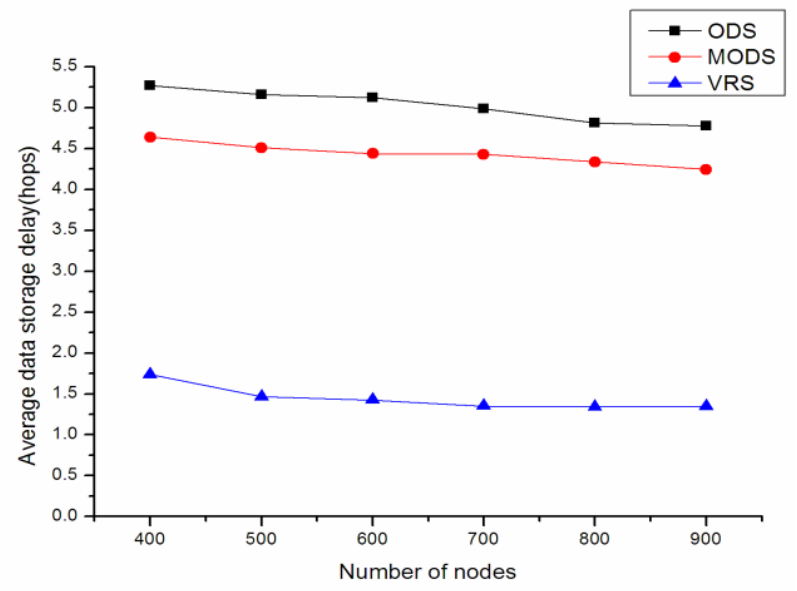

Figure 5. Average Data Storage Delay

In this section, we test the performance of the average delay of data storage and retrieval. The delay of the event data storage is represented as the hop count that the event data travels before it rests on some nodes. The delay of the event data retrieval is represented as the hop count that the query travels from the consumer to the meeting point plus the hop count that the query result travels from the meeting point to the consumer. We set the initial energy to $3 \mathrm{~J}$ in this test.

Figure5 shows the average delay of the event data storage in ODS, MODS and VRS when the data effective time is set to $1 \mathrm{~s}$. In fact, it does not matter what value the data effective time is set to when testing the performance of the delay of the event data storage, because the data effective time does not affect the routing track of the event data at all. We can see that the average delay of the event data storage in VRS is much less than that 
in ODS and MODS. This is because, in VRS, the event data only need to be routed into the rendezvous ring, and this is much easier than routing the event data to a rendezvous node. Besides, there are many nodes that are located in the virtual ring, and they just store the data they generated locally. This decreases the average delay of the event data storage greatly. By the way, the average delay of the event data storage in MODS is less than that in ODS, because there are many rendezvous nodes in MODS, and each producer only needs to choose the nearest rendezvous node to store the event data.

Figure6 demonstrates the average delay of the event data retrieval in ODS, MODS and VRS with different data effective time. We can see that, as the data effective time decreases, the average delay of the event data retrieval increase in the three schemes. This is because, when the data effective time decreases, the query has to travel much longer to meet the desired event data. Note that, the average delay of the event data retrieval in MODS is more than that in ODS at the beginning, and becomes less than that in ODS as the node number increase to a certain number. The reason is that, when the node number is not big enough, many queries in MODS have to access many rendezvous nodes to find the desired event data, under the given event data generating rate in our experiment. However, when the node number becomes large enough, the probability of finding the desired event data before arriving at the nearest rendezvous node arises. Thus, the average delay of the data retrieval in MODS becomes less than that in ODS when the node number increases to a certain number.

Figure6 also clearly shows that the average delay of the event data retrieval in VRS is less than that in ODS and MODS. This is because, there are many nodes that are located in the virtual ring, and they discover the desired event data by broadcasting queries to their neighbors. The probability is very high to meet the desired event data in a short hop count in such a way. Besides, the nodes that are located outside the rendezvous ring are much easier to route the queries to the rendezvous ring in VRS than most of the nodes in ODS and MODS to route the queries to the rendezvous nodes.

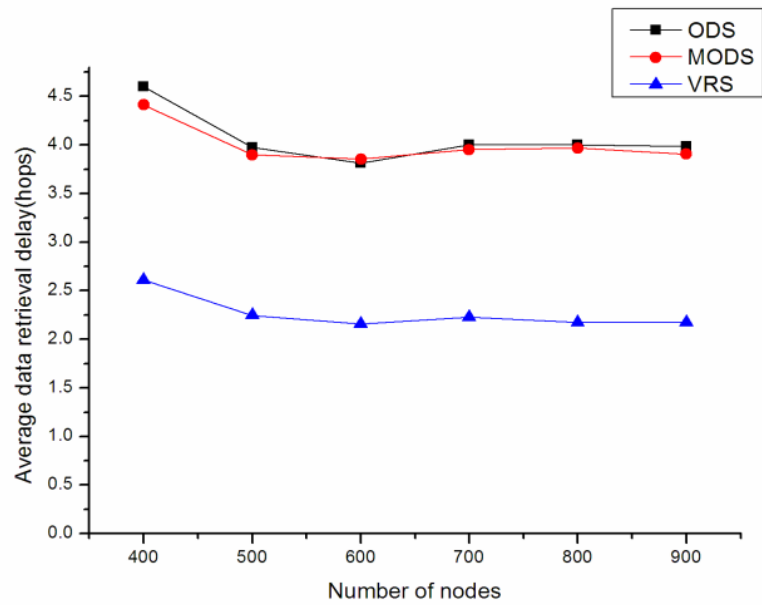

(a) 


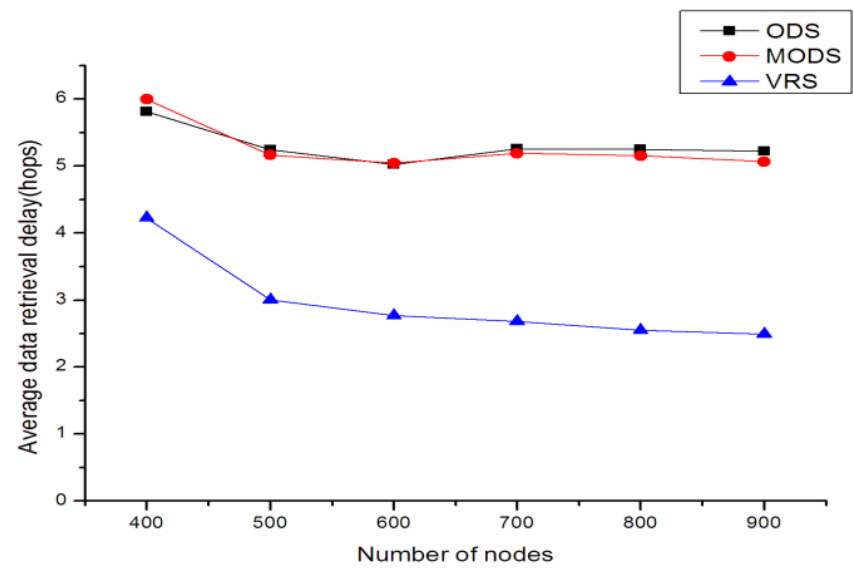

(b)

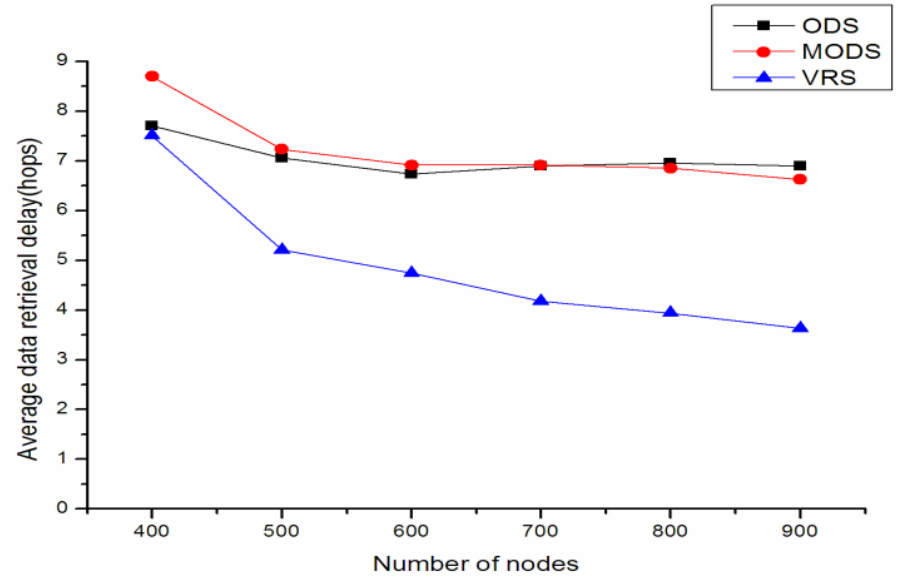

(c)

Figure 6. Average Delay of Data Retrieval with Increasing Node Number: (a) $\tau=1 \mathrm{~s}$; (b) $\tau=0.5 \mathrm{~s}$; (c) $\tau=0.2 \mathrm{~s}$

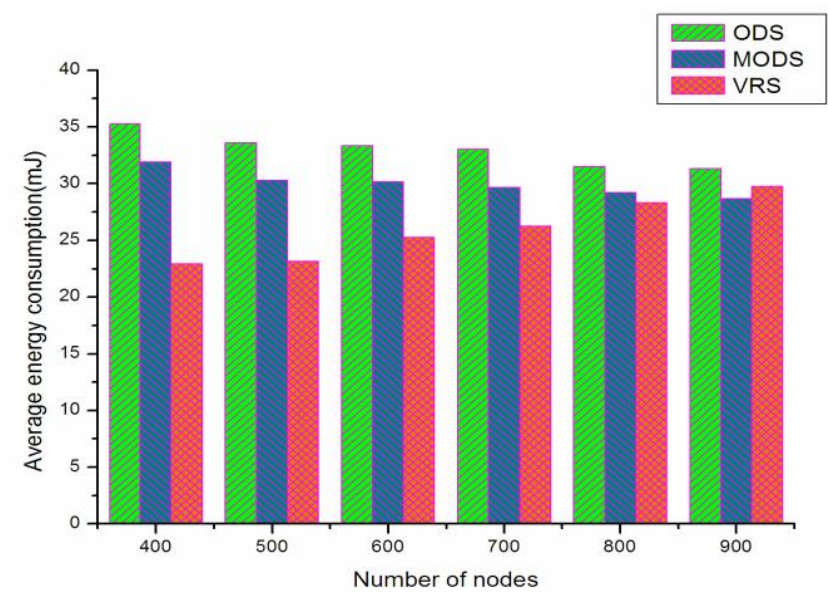

Figure 7. Average Energy Consumption of Each Sensor Node

\subsection{Energy Consumption and Load Balancing}

This experiment tests the performance of ODS, MODS and VRS on the average energy consumption and the load balancing. The initial energy of each sensor node is set to $3 \mathrm{~J}$, and the data effective time is set to $1 \mathrm{~s}$. 
Figure 7 shows the average energy consumption of the each sensor node in the three schemes. Although the average hop count of data storage and retrieval in VRS is much less than that in ODS and MODS, the queries that arrive at the rendezvous ring need to be broadcasted to discover the desired data, and this consumes a lot of energy. Thus, the average energy consumption in VRS is not so much less than that in ODS and MODS, and they are even very close when the node number increases to a certain number.

Figure 8 below shows the energy consumption of the node that consumes the most energy among all the nodes in the sensor network. The energy consumption by the node that consumes the most energy in ODS is much higher than that in MODS and VRS. This is because, theoretically speaking, the node that consumes the most energy must be a rendezvous node or a node in the rendezvous ring. There is only one rendezvous node in ODS, and the rendezvous node have to receive all the event data coming from all the producers. As the number of rendezvous nodes increases, the load of the rendezvous nodes will be reduced.

The energy consumption of each sensor node in the three schemes is shown in Figure 9. It is very clear that VRS balances the load much better than ODS and MODS.

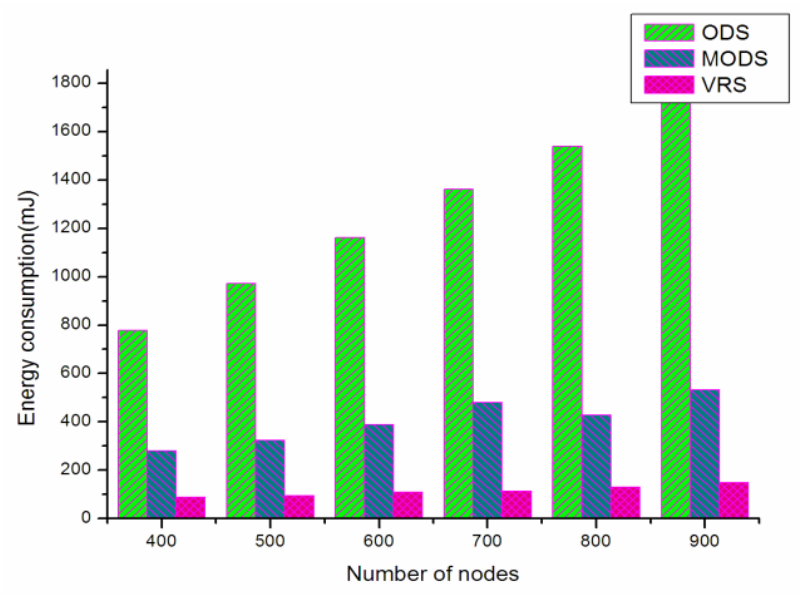

Figure 8. Energy Consumption of the Node with the Most Energy Consumption

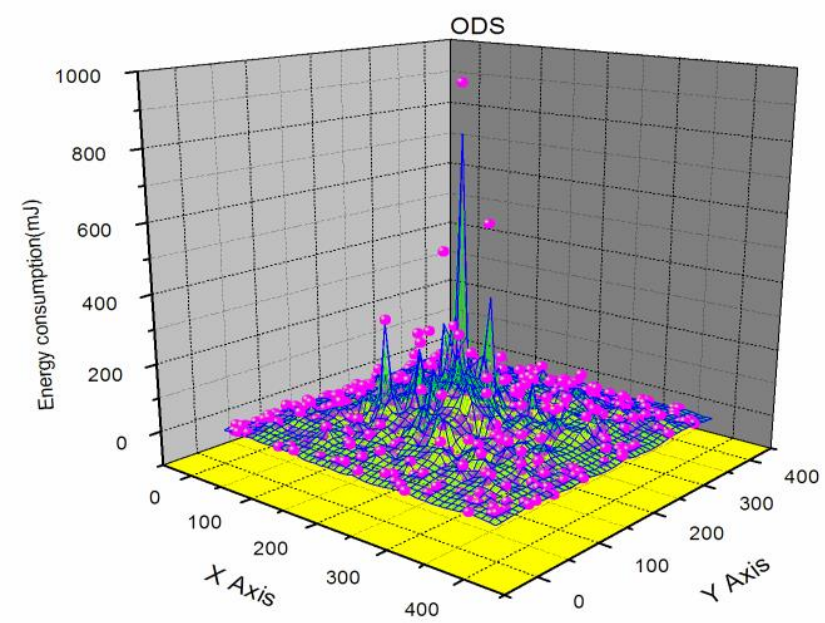

(a) 


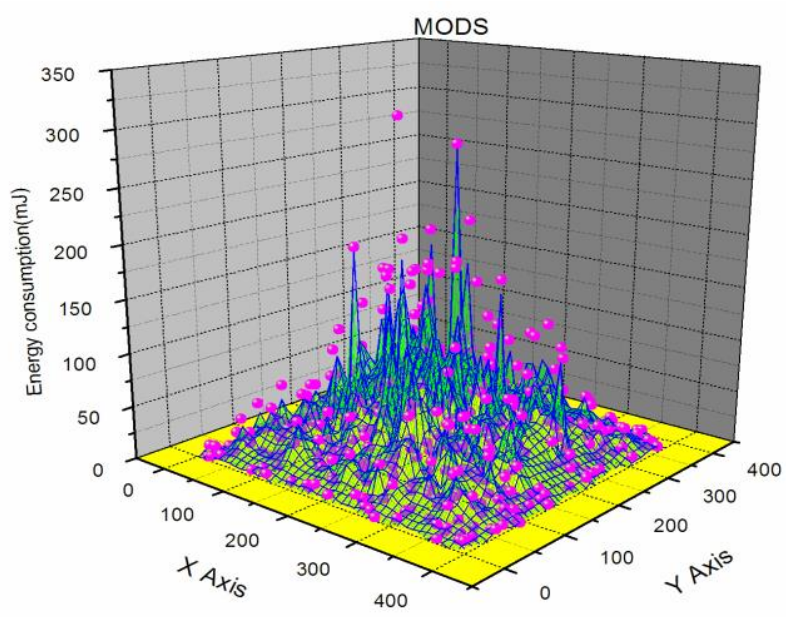

(b)

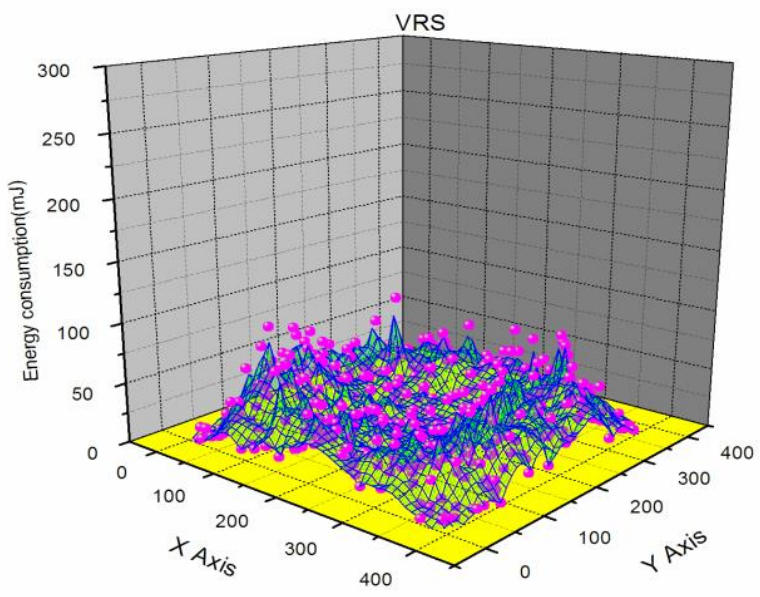

(c)

Figure 9. Distribution of Energy Consumption: (a) ODS; (b) MODS; (c) VRS

\section{Conclusion}

In this paper, we study the information-brokerage problem, and compare the three schemes ODS, MODS and VRS through extensive simulation. Among the three schemes, VRS is our former work, and ODS and MODS are two schemes which were presented before and after the time when VRS was proposed respectively by other researchers. We add two more important algorithms in this paper to make VRS more perfect. We only compare the three schemes because, as far as we know, few schemes except the abovementioned three schemes considering the change of both the data generating rates of the producers and the query frequencies of the consumers when solving the informationbrokerage problem. By comparing and analyzing the simulation results, we find that VRS does perform much better than MODS and ODS on network life time, the delay of data storage, and the delay of retrieval as well as load balancing. Although the difference between VRS and ODS on the total energy consumption of data storage and retrieval is very small, VRS is still a much better choice to solve the information-brokerage problem in wireless sensor networks.

\section{Acknowledgments}

This research is supported by NSFC (Natural Science Foundation of P. R. China, No. 61562005), the Science and Technology Project of Henan Province (NO.172102210454), 
the Natural Science Foundation of Henan Province of P. R. China (162300410234), the Nanhu Scholars Program for Young Scholars of XYNU, and the supporting program of young backbone teachers in Xinyang Normal University in Henan Province of P. R. China.

\section{References}

[1] S. Scott, R. Sylvia, K. Brad, G. Ramesh, and E. Deborah, "Data-centric storage in sernsornets", ACM SIGCOMM Computer Communication Review,vol. 33, no. 1, (2003), pp. 137-142.

[2] R. Sylvia, K. Brad, S. Scott, E. Deborah, R. Govindan, L. Yin, and F. Yu, "Data-centric storage in sensornets with GHT, a geographic hash table", Mobile Networks and Applications, vol. 8, no. 4, (2003), pp. 427-442.

[3] M. Xingpo M., G. Jianliang, W. Weiping, and W. Jianxin, "A Virtual-Ring-Based Data Storage and Retrieval Scheme in Wireless Sensor Networks", International Journal of Distributed Sensor Networks, (2012), pp.1-10. doi:10.1155/2012/763015.

[4] Z. C. Yu, B. Xiao, and S. Zhou, "Achieving optimal data storage position in wireless sensor networks", Computer Communications, vol. 33, no. 1, (2010), pp. 92-102.

[5] L. Zhu, W. Gao, J. Hong, and X. Deng, "Adaptive information brokerage of multiple storage nodes in optical sensor networks", Optik- International Journal for Light and Electron Optics, vol. 126, no. 23, (2015), pp. 4358-4361.

[6] C. Intanagonwiwat, R. Govindanj, and D. Estrin, "Directed diffusion: A scalable and robust communication paradigm for sensor networks", Proceedings of the 6th Annual Int'l Conferenceon Mobile Computing and Networking, Boston, MA, USA, (2000) August 06-11.

[7] S. Shenker, S. Ratnasamy, B. Karp, R. Govindan, and D. Estrin, "Data-centric storage in sensor nets", SIGCOMM Comput. Commun. Rev., vol. 33, no. 1, (2003), pp. 137-142.

[8] S. Madden, M. J. Franklin, J. M. Hellerstein, and W. Hong, "TAG:A tiny aggregation service for ad-hoc sensor networks", Proceedings of the 5th Symp.on Operating System Design and Implementation, Boston, MA, USA, (2002) December 9-11.

[9] D. Braginsky, and D. Estrin, "Rumor routing algorithm for sensor networks", Proceedings of the First ACM International Workshop on Wireless Sensor Networks and Applications, Atlanta, Georgia, USA, (2002) September 28-28.

[10] S. Ratnasamy, B. Karp, L. Yin, F. Yu, D. Estrin, R. Govindan, and S. Shenker, "Ght: a geographic hash table for data-centric storage", Proceedings of the First ACM International Workshop on Wireless Sensor Networks and Applications, Atlanta, Georgia, USA, (2002) September 28-28.

[11] Y. X.. Lai, H. Chen, and Y. F. Wang, "Dynamic balanced storage in wireless sensor networks", Proceedings of the 4th Workshop on Data Management for Sensor Networks, University of Vienna, Austria, (2007) September 23-28.

[12] B. Ratnabali, C. Kaushik, P. A. Dharma, "Attribute allocation and retrieval scheme for large-scale sensor networks", International Journal of Wireless Information Networks, vol. 13, no. 4, (2006), pp. 303-315.

[13] W. H. Liao, and W. C. Wu, "Effective hotspot storage management schemes in wireless sensor networks", Computer Communications, vol. 3, no. 10, (2008), pp. 2131-2141.

[14] K. T. Ma, K. Y. Cheng, K. S. Liu, and T. Vincent, "Improving data centric storage with diffuse caching in wireless sensor networks", Wirel. Commun. Mob. Comput., vol. 9, no. 3, (2009), pp. 347-356.

[15] L. Hung-Cuong,H. uyennet, and N. Zerhouni, "Mobile Effect Reduction in Data-Centric Storage for Wireless Sensor Networks", Proceedings of the 3rd IET International Conference on Intelligent Environments, the University of Ulm, Germany, (2007) September 24 -25.

[16] P. Junho, S. Dongook, K. Hyunju, P. Kisoon, L. Byoungyup, and Y. Jaesoo, "A data-centric storage scheme for high storage utilization in wireless sensor networks", Cluster Comput., vol. 18, no. 1, (2015), pp. 247-257.

[17] R. Sarkar, X. Zhu, and J. Gao, "Double Rulings for Information Brokerage in Sensor Networks", Proceedings of the 12th Annual International Conference on Mobile Computing and Networking, Los Angeles, CA, USA, (2006) September 24-29.

[18] Q. Fang, J. Gao, and L. J. Guibas, "Landmark- based information storage and retrieval in sensor Networks", Proceedings of the 25th IEEE International Conference on Computer Communications, Barcelona, Spain, (2006) April 23-29.

[19] C. Damdinsuren, D. Kominami, M. Sugano., M. Murata, T. Hatauchi, "Lifetime extension based on residual energy for receiver-driven multi-hop wireless network”, Clust. Comput., vol. 16, no. 3, (2013), pp. 469-480.

[20] S. Xie and Y. Wang, "Construction of Tree Network with Limited Delivery Latency in Homogeneous Wireless Sensor Networks", Wireless Personal Communications, vol. 78, no. 1, (2014), pp.231-246.

[21] B. Karp, and H. T. Kung, "GPSR: Greedy perimeter stateless routing for wireless networks", Proceedings of the 6th Annual ACM/IEEE Int'1 Conf. on Mobile Computing and Networking, Boston, MA, USA, (2000) August 6-11. 
[22] J. Shen, H. Tan, J. Wang, J. Wang, and S. Lee, "A Novel Routing Protocol Providing Good Transmission Reliability in Underwater Sensor Networks", Journal of Internet Technology, vol. 16, no. 1, (2015), pp. 171-178.

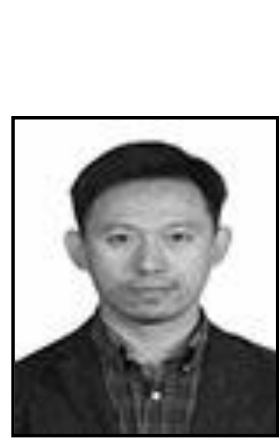

\section{Authors}

Xing-Po Ma, received his $\mathrm{PhD}$ degree in computer application technology from Central South University in China in 2013. Since $1^{\text {st }}$ July 2014 , he has been working in Xinyang Normal University in China. He was awarded the title of the youth backbone teacher by Xinyang Normal University in 2015. He is now the member of Chinese Association of Automation. His research interests include 5G network and IOT.

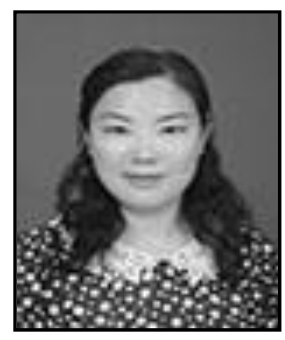

Song Guo, received the MSc degree in computer applications technology from Wuhan University of Technology University, China in 2005. She is currently a professor with Xinyang Normal University. Her research interests include computer networks, Internet of Things and services-oriented architecture.

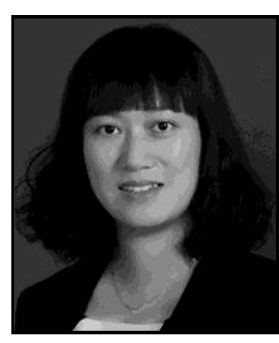

Lei $\mathbf{L i}$, received her MSc degree in computer science from China University of Geosciences in 2007. She is an associate professor at Xinyang Normal University. Her research interests include computer network, wireless sensor networks.

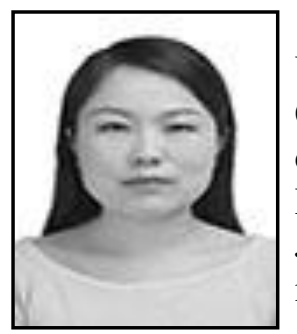

Yan-Ge Sun, received her BSc degree from the Xinyang Normal University (XYNU), Xinyang, in 2004 and the MSc degree from the Central China Normal University (CCNU), Wuhan, in 2007, both in computer science. She is currently pursuing the Ph.D. degree with the Department of Electrical and Computer Engineering, Beijing Jiaotong University. Her research interests include data mining and machine learning.

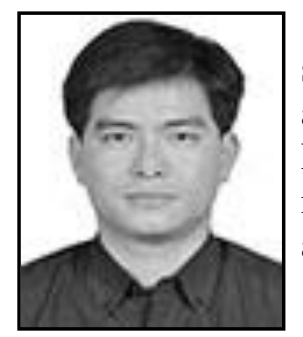

Jun-Bin Liang, received the BSc and MSc degrees in computer science from Guangxi University, in 2000 and 2005, respectively, and the $\mathrm{PhD}$ degree from Central South University, China in 2010. $\mathrm{He}$ is currently a professor in Guangxi University. His research interests include mobile ad hoc networks, wireless sensor networks and distribued systems. 
International Journal of Grid and Distributed Computing

Vol. 10, No. 5 (2017) 